\title{
Recent $\mathrm{H}$ majority inverted radio frequency heating scheme experiments in JET-ILW
}

\author{
${ }^{1}$ LPP-ERM/KMS, EUROfusion Consortium Member - Trilateral Euregio Cluster, Brussels, Belgium \\ ${ }^{2}$ CCFE, Culham Science Centre, Abingdon, OX14 3DB, UK \\ ${ }^{3}$ Max-Planck-Institut für Plasmaphysik, Garching, Germany \\ ${ }^{4}$ Department of Applied Physics, Ghent University, Ghent, Belgium \\ ${ }^{5}$ Institute of Plasma Physics and Laser Microfusion, Hery 23, 01-497 Warsaw, Poland \\ ${ }^{6}$ CEA, IRFM, F-13108 Saint-Paul-lez-Durance, France \\ ${ }^{7}$ Department of Physics and Astronomy, Uppsala University, SE-75120 Uppsala, Sweden \\ ${ }^{8}$ Istituto di Fisica del Plasma "Piero Caldirola", IFP-CNR, via R. Cozzi 53, 20125 Milano, Italy \\ ${ }^{9}$ NERSC, MS 59R4010A Berkeley, CA 94720, USA \\ ${ }^{10}$ Associação EURATOM/IST, Instituto de Plasmas e Fusão Nuclear, Instituto Superior \\ ${ }^{11}$ Dipartimento di Fisica "G. Occhialini", Università di Milano-Bicocca, 20126 Milano, Italy \\ ${ }^{12}$ VTT Technical Research Centre, FI-02044 Espoo, Finland \\ ${ }^{13}$ Technical University of Denmark, Department of Physics, Kgs. Lyngby, Denmark \\ ${ }^{14}$ ITER Organization, Route de Vinon-sur-Verdon, CS 90 046, 13067 St. Paul-lez-Durance Cedex, France \\ ${ }^{15}$ Ecole Polytechnique Fédérale de Lausanne (EPFL), SPC, CH-1015 Lausanne, Switzerland
}

Dirk Van Eester ${ }^{1, *}$, Ernesto Lerche ${ }^{1,2}$, Yevgen Kazakov ${ }^{1}$, Philippe Jacquet ${ }^{2}$, Yuri Baranov ${ }^{2}$, Volodymyr Bobkov ${ }^{3}$, Kristel Crombé $^{1,4}$, Agata Czarnecka ${ }^{5}$, Remi Dumont ${ }^{6}$, Pierre Dumortier ${ }^{1,2}$, Jacob Eriksson ${ }^{7}$, Luca Giacomelli $^{8}$, Carine Giroud ${ }^{2}$, Marc Goniche ${ }^{6}$, Carl Hellesen ${ }^{7}$, Vasily Kiptily ${ }^{2}$, Natalia Krawczyk ${ }^{5}$, Tuomas Koskela ${ }^{9}$, Filomena Nave ${ }^{10,2}$, Massimo Nocente $^{11}$, Jef Ongena ${ }^{1}$, Marko Santala ${ }^{2,12}$, Mirko Salewski ${ }^{13}$, Mireille Schneider ${ }^{14}$, Henri Weisen ${ }^{15}$ and JET contributors ${ }^{\mathrm{x}}$

${ }^{\mathrm{x}}$ See the author list of "Overview of the JET results in support to ITER" by X. Litaudon et al. to be published in Nuclear Fusion special issue: "Overview and summary reports from the $26^{\text {th }}$ Fusion Energy Conference (Kyoto, Japan, 17-22 October 2016)"

\begin{abstract}
Inverted ${ }^{3} \mathrm{He}$ and D ion cyclotron minority heating scenarios were recently tested in JET-ILW. They confirm the good heating efficiency at low concentrations of $\sim 3 \%$. The ${ }^{3} \mathrm{He}$ minority heating scheme is only modestly affected by the change from a carbon (JET-C) to a Beryllium (JET-ILW) wall but unlike what was the case in JET-C, the intrinsic Be ions D-like particles in terms of charge-over-mass ratio do not prevent the $\mathrm{D}$ ( or ${ }^{4} \mathrm{He}$ ) minority regime from being exploited. Direct and indirect evidence of the existence of fast particle subpopulations was found in both cases.
\end{abstract}

\section{Introduction}

Lacking other ICRF (Ion Cyclotron Resonance Frequency) heating options at full field, ${ }^{3} \mathrm{He}$ and ${ }^{4} \mathrm{He}$ minority fundamental cyclotron radio frequency heating schemes will be crucial in ITER's non-activated $\mathrm{H}$ majority phase.

Both these so-called "inverted" schemes can be accessed in JET. They were recently tested in presence of the ITER-like wall (ILW). For "inverted" schemes the charge-over-mass ratio of the majority is larger than that of the minority so that the confluence layer is in between the antenna and the minority ion cyclotron layer where the dominant heating occurs. In these schemes, the mode conversion regime is reached at much lower minority concentration $(2-3 \%)$ than is the case of standard schemes, for which $(\mathrm{Z} / \mathrm{A})_{\min }>(\mathrm{Z} / \mathrm{A})_{\text {maj; }}$; $\mathrm{Z}$ is the charge and $\mathrm{A}$ the atomic number. Moreover, increasing the minority concentration shifts the mode conversion layer where the Bernstein or ion cyclotron wave are born towards the low rather than the high magnetic field side with respect to the ion-cyclotron absorption layer.

Both in the past with the $\mathrm{C}$ wall and more recently with the ITER-like wall, fundamental ${ }^{3} \mathrm{He}$ heating has been successfully exploited in JET [1-3]. It was used in the 2016 experimental campaign for $\mathrm{L}-\mathrm{H}$ threshold experiments [4] and transport studies [5]. For the former, the available power was marginally sufficient to reach $\mathrm{H}$ mode at high field. For the latter, exploiting the minority heating regime at modest concentration and the mode conversion regime at high concentration allowed providing localized core ion and electron heat sources, respectively.

Equipped with a $\mathrm{C}$ wall in the past, JET's plasmas typically contained $\sim 2 \%$ of $\mathrm{C}$, the presence of which excluded fundamental minority ${ }^{4} \mathrm{He}$ or $\mathrm{D}$ heating in $\mathrm{H}$ plasmas, the mode conversion regime being reached immediately as $(\mathrm{Z} / \mathrm{A})_{\mathrm{D}}=(\mathrm{Z} / \mathrm{A})_{4_{\mathrm{He}}}=(\mathrm{Z} / \mathrm{A})_{\mathrm{C}} \quad[1]$. Because JET's walls are now made of Be rather than $\mathrm{C}$, these inverted minority heating regimes can now be accessed and heating schemes re-assessed. The fundamental D minority ICRH case was briefly revisited in the last JET-ILW hydrogen campaign; some representative findings will be presented.

\section{Experimental results}

The ${ }^{3} \mathrm{He}$ minority experiments were conducted at $\mathrm{B}_{0}=3.2 \mathrm{~T}$ to absorb waves at the fundamental cyclotron resonance layer of ${ }^{3} \mathrm{He}$. This layer resides in the hot plasma core when using $\mathrm{f}=32.5 \mathrm{MHz}$, a frequency in reach both for the A2 [6] and the ILA antenna [7,8]. Up to $7.6 \mathrm{MW}$ was launched into the plasma. Dipole phasing

\footnotetext{
${ }^{*}$ Corresponding author: d.van.eester@fz-juelich.de
} 
was imposed. The plasma was in L-mode and the plasma current was $\mathrm{I}_{\mathrm{p}}=1.8 \mathrm{MA}$; the strike point was on divertor tile 5 .

Figure 1 shows the temporal evolution of some key parameters while Fig. 2 gives an example of the density and temperature profiles. Hydrogen was the majority ion species and the central electron density was $\mathrm{N}_{\mathrm{eo}}=4 \times 10^{19} \mathrm{~m}^{-3}$. The (hydrogen) neutral beam power was modest, $\mathrm{P}_{\mathrm{NBI}} \sim 3 \mathrm{MW}$. About $30 \%$ of the total power is lost through radiation. Temperatures of up to $4 \mathrm{keV}$ were reached, the electron temperature typically exceeding that of the ions in spite of the presence of sawteeth; opposite to past experiments performed at higher current $\left(I_{p}=2.45 \mathrm{MA}\right)$, the recovery from sawtooth crashes was prompt and significant [9]. Relying on break-in-slope analysis of the plasma energy, optimal overall heating was observed at ${ }^{3} \mathrm{He}$ minority concentrations of $2-4 \%$; see Fig. 3. Heating efficiencies of $\mathrm{P}_{\mathrm{abs}} / \mathrm{P}_{\mathrm{in}} \sim 70 \%$ were reached. The large spread in the efficiency at a given ${ }^{3} \mathrm{He}$ concentration is due to the variation of the parameters (sawtooth behavior, density profile, current, ...). The plasma energy crudely increases by $\sim 0.1 \mathrm{MJ} / \mathrm{MW}$, which is a factor of $\sim 2$ lower than the performance for the frequently used $\mathrm{H}$ minority in a D majority JET ICRH scheme [10]; this lower efficiency is largely brought about by the different confinement of D plasmas but is also to a lesser extent due to the actual heating performance. At too small minority concentration the heating degrades while at larger concentrations the mode conversion regime is entered and direct electron heating comes more significantly into play. The actual mode conversion regime - where direct electron damping clearly dominates ion damping - was not reached. At the small minority concentrations typical for guaranteeing minority heating in inverted scenarios, the fraction of power indirectly flowing to the electrons via Coulomb collisional relaxation of the minority is significant. For the parameters relevant for the experiment, the tipping point where bulk ions collisionally receive as much energy as the electrons is at about $X\left[{ }^{3} \mathrm{He}\right]=\mathrm{N}_{3}{ }_{\mathrm{He}} / \mathrm{N}_{\mathrm{e}}=1.5 \%$ when $P_{R F}=0.5 \mathrm{MW} / \mathrm{m}^{3}$. This explains why the experimentally observed core electron heating efficiency electron response is significant except when going to too high concentrations at which point the mode conversion efficiency response for significant direct core electron heating is lost and waves are sloshing over the plasma multiple times before being fully absorbed [3].

At the low concentrations optimal for heating ions when relying on an inverted scheme, energetic tails are formed. Consistent with the presence of the formation of fast particle ion populations, sawtooth stabilization is observed. A clear response of the sawtooth frequency to a step in ICRH power is observed. At $\mathrm{X}\left[{ }^{3} \mathrm{He}\right] \sim 2 \%$ the sawteeth are fully stabilised.

Aside from assessing the overall heating performance, JET is equipped with diagnostics allowing to diagnose in detail the response of both electrons and bulk ions.
Figure 4 shows an example of the experimental power deposition profiles obtained by modulating the ICRH power and analysing it performing a Fourier analysis of the response of the electron temperature to the changes of the power. When focusing on electron dynamics a modulation frequency $f_{\text {mod }}$ is preferred $\left(f_{\text {mod }}=25 \mathrm{~Hz}\right.$ for the depicted data); focussing on the slower ion dynamics (not done here) necessitates accounting for the nonlinear saturation of the ion temperature response and requires operating at smaller modulation frequencies (see e.g. [11] with $\left.\mathrm{f}_{\text {mod }} \sim 4 \mathrm{~Hz}\right)$. At low ${ }^{3} \mathrm{He}$ concentrations, the deposition is close to the centre and highly peaked. When the concentration increases, the peak of the power deposition moves outward, reflecting the moving of the ion-ion hybrid layer whose position critically depends on the minority concentration and at which efficient localised absorption of the Bernstein wave by electrons takes place [12]. When $\mathrm{X}\left[{ }^{3} \mathrm{He}\right] \sim 10 \%$, the maximum of the power deposition has dropped by a factor 6 and its location has shifted to half-radius.

Adding a small minority of ${ }^{4} \mathrm{He}$ to a $\mathrm{H}$ plasma allows to study a second inverted heating scheme relevant for the initial phase of ITER. ${ }^{4} \mathrm{He}$ and D sharing the same massto-charge ratio, ICRH waves respond similarly to them. When JET was equipped with a $\mathrm{C}$ wall, $\mathrm{D}$ minority heating experiments were unsuccesful since $\mathrm{C}$ - equally sharing the deuteron's Z/A - eroded from the wall contaminated the main plasma and increased the D-like ion population concentration well beyond the limit tolerable for promising minority heating [1]. The $\mathrm{C}$ being replaced by $\mathrm{Be}$ in the ITER-like wall [13], this problem no longer poses and hence the scheme was readdressed when the $\mathrm{D}$ concentration was very low (X[D] 0.7\%); X[Be] was estimated to be of order $0.5 \%$. Reaching the $\mathrm{D}$ minority heating regime at $\mathrm{B}_{0}=3.33 \mathrm{~T}$ requires lowering the driver frequency to $25.3 \mathrm{MHz}$, beyond the reach of the ILA. The A2 antenna's - on the other hand - can be operated at that frequency, be it that the system is not designed for it so that only limited power $\left(\mathrm{P}_{\mathrm{RF}} \sim 3 \mathrm{MW}\right)$ can be launched. $\mathrm{D}$ minority heating was briefly tested in the recent campaign; ${ }^{4} \mathrm{He}$ minority heating experiments were postponed to later.

The obtained heating efficiencies were somewhat lower than those obtained for ${ }^{3} \mathrm{He}\left(\mathrm{P}_{\mathrm{abs}} / \mathrm{P}_{\mathrm{in}} \sim 50 \%\right)$; the D levels remained below the level $\mathrm{X}[\mathrm{D}] \sim 2 \%$ required for optimal heating. Energy and temperature increases of $0.08 \mathrm{MJ} / \mathrm{MW}$ and $0.3 \mathrm{keV} / \mathrm{MW}$, respectively, were seen. Evidence of fast D was clearly observed by various diagnostics while such evidence was not found in presence of the $\mathrm{C}$ wall. Tail temperatures of $200 \mathrm{keV}$ were confirmed by the neutral particle analyser as well the time-of-flight diagnostics relying on neutrons born from the D-D reaction. The neutron rate clearly - but nonlinearly - correlates with the ICRH power. And gamma ray detectors [14] testify for nuclear reactions necessitating a fast $\mathrm{D}$ population. More details can be found in [15]. 
Figure 5 depicts the counts per energy bin of the neutrons detected by the time-of-flight neutron detector; velocity being inversely proportional to time, evidence for fast particles is on the left in the plot. The black dots are the data while the lines are the corresponding error bars. The blue line is a synthetic diagnostic fit based on a Maxwellian deuterium distribution, which has an anisotropic pitch angle distribution centered closely around $90^{\circ}$, as expected for the RF accelerated ions. The dotted vertical line delimits the position beyond which JET's beams do not have any counts, illustrating that the RF wave's capacity to produce high energy deuterons; neutral beam injection is absent in the depicted shot.

Dedicated experiments were also performed to study D minority NBI heating [16]. Aside from operating at somewhat higher concentrations, reducing the plasma's collisionality and widening the cyclotron damping region is known to be beneficial (see e.g. [17] on the synergistic effects of combining ICRH and NBI). On top of that " classical» effect most often exploited for boosting harmonic heating schemes [18] and referred to as « NBI preheating ", a supplementary heating scenario has been proposed more recently and was experimentally tested on JET and Alcator C-Mod [19-21]. It profits from the presence of 2 ion species ( $\mathrm{H}$ and $\mathrm{D}$ in this case) to align the cyclotron layer of a third ion species $\left({ }^{3} \mathrm{He}\right)$ with a region where the polarisation is locally modified to make it well suited for ion heating. Combining assets from various effects makes RF heating of a minority beam playing the role of the third type of ions species as its dominant cyclotron damping region is Doppler shifted away from the cold plasma cyclotron layer - an efficient heating scheme.

\section{Summary and discussion}

Inverted ${ }^{3} \mathrm{He}$ and D ICRF heating scenarios in hydrogen majority plasmas were recently tested on JET-ILW at $\mathrm{B}_{\mathrm{o}} \sim 3.3 \mathrm{~T}$. Both are viable options for the non-active fullfield operation of ITER $\left(B_{0}=5.3 T\right)$ at respectively 53 and $40 \mathrm{MHz}$ [22].

Heating efficiencies of $\sim 70 \%$ have been achieved with ${ }^{3} \mathrm{He}$ minority heating and and the experimental findings are in line with theoretical expectations. The change from JET-C to JET-ILW has limited impact on this minority heating scenario. Due to the optimal concentration being small $(\sim 3 \%)$, fast ion tails are created and Coulomb collisional electron heating is dominant in these conditions. This was confirmed by fast particle measurements, evidence of sawtooth stabilization and by the study of the electron response to ICRF power modulation. Real time control of $\mathrm{X}\left[{ }^{3} \mathrm{He}\right]$ is important in these conditions to keep the RF wave absorption in the optimal regime.

Whereas in JET-C carbon eroded from the JET walls prevented exploiting the $\mathrm{D}$ or ${ }^{4} \mathrm{He}$ minority heating schemes $\left(\mathrm{C}\right.$ being present at $\sim 2 \%$ levels and $(\mathrm{Z} / \mathrm{A})_{\mathrm{C}}$ being identical to that of $\mathrm{D}$ and ${ }^{4} \mathrm{He}$ so that the effective D-like concentration was too high for minority heating), proof-of-principle experiments in JET-ILW demonstrated successful D ion heating although optimal $\mathrm{D}$ minority concentrations were not reached. Evidence of the formation of fast particle subpopulations is available for this scenario as well and suggests that $\mathrm{D}$ ion tails with energies $\sim 200 \mathrm{keV}$ have been created.

$\mathrm{N}=1{ }^{3} \mathrm{He}$ minority heating in $\mathrm{H}$ proves to be a promising candidate for ITER's full field non-activated phase. Dedicated experiments are required to document the potential of the $\mathrm{N}=1{ }^{4} \mathrm{He}$ minority heating scheme but the first evidence using a $\mathrm{D}$ minority $\left(\mathrm{Z} / \mathrm{A}_{\mathrm{D}}=\mathrm{Z} / \mathrm{A}_{4}{ }_{\mathrm{He}}\right)$ is encouraging.

\section{Acknowledgements}

This work has been carried out within the framework of the EUROfusion Consortium and has received funding from the Euratom research and training programme 2014-2018 under grant agreement No 633053. The views and opinions expressed herein do not necessarily reflect those of the European Commission. The views and opinions expressed herein do not necessarily reflect those of the ITER Organization.

\section{References}

1. M.-L. Mayoral et al., Nucl. Fusion 46, S550-S563 (2006)

2. P.U. Lamalle et al, Nucl. Fusion 46, 391 (2006)

3. D. Van Eester et al, Plasma Phys. Contr. Fusion 54, 074009 (2012)

4. J. Hillesheim et al., Proc. 26 $6^{\text {th }}$ IAEA-FEC Conference, Kyoto (2016), EX/5-2

5. P. Mantica et al., Proc. $26^{\text {th }}$ IAEA-FEC Conference, Kyoto (2016), EX/P6-14

6. A. Kaye et al., Fusion Engineering and Design, 24, 1-21 (1994)

7. F. Durodié et al. , Fusion Engineering and Design 84, Issues 2-6, 279-283 (2009)

8. F. Durodié et al., Proc. 29th SOFT conference, Prague, P4.037 (2016)

9. D. Van Eester et al., Proc. $42^{\text {nd }}$ EPS Conf. on Plasma Physics (Lisbon, 2015), P2.117

10. V. P. Bhatnagar et al., Plasma Phys. Contr. Fusion 22, 99 (1991)

11. F. Ryter et al., Plasma Phys. Contr. Fusion 51, 113016 (2011)

12. R. Perkins, Nuclear Fusion 17, 1197 (1977)

13. R. Neu et al., Plasma Phys. Control. Fusion 44, 811 (2002)

14. Kiptily V G et al 2002 Nucl. Fusion 42 999-1007

15. E.A. Lerche et al., in preparation for Plasma Phys. Contr. Fusion 
16. J. Ongena et al., "Observations of Synergetic Acceleration of D-NBI Ions in the Vicinity of the Mode Conversion Layer in H-D Plasmas", invited talk at this conference.

17. R. Koch et al., Fus. Science Technology 47, 97 (2005)

18. M. Schneider et al., Nucl. Fusion 56, 112022 (2016)

19. Ye. O. Kazakov et al., Nucl. Fusion 55, 032001 (2015)

20. Ye. O. Kazakov et al., Efficient generation of energetic ions in multi-ion plasmas by radiofrequency heating, Nature Physics (2017); http://dx.doi.org/10.1038/nphys4167

21. J. Wright et al., Proc. 26th IAEA Fusion Energy Conference, Kyoto, EX/P3-5 (2016)

22. M. Schneider et al., "ICRH scenarios for the ITER non-active phase", paper C-19 at this conference.

23. M. G. Johnson et al., Nucl. Instrum. and Methods in Physics Research A, 591, 417-430 (2008)

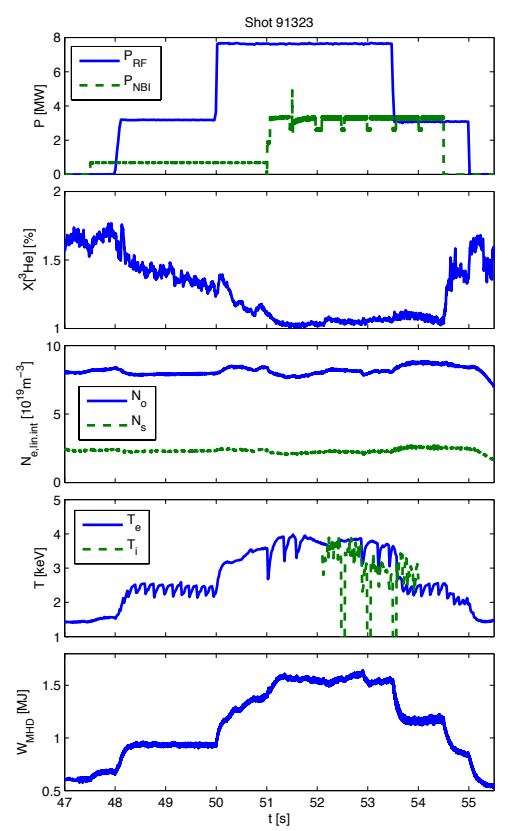

Fig. 1 : RF and NBI power, minority concentration, core and edge line integrated densities, electron and ion temperature and plasma energy for the ${ }^{3} \mathrm{He}$ minority heating study.

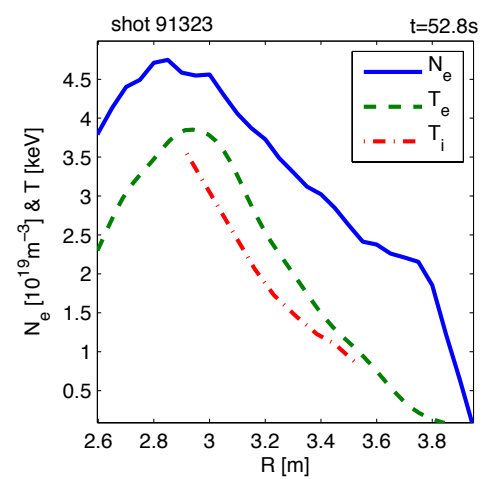

Fig. 2 : Density and temperature profiles of the $91323{ }^{3} \mathrm{He}$ minority heating shot.

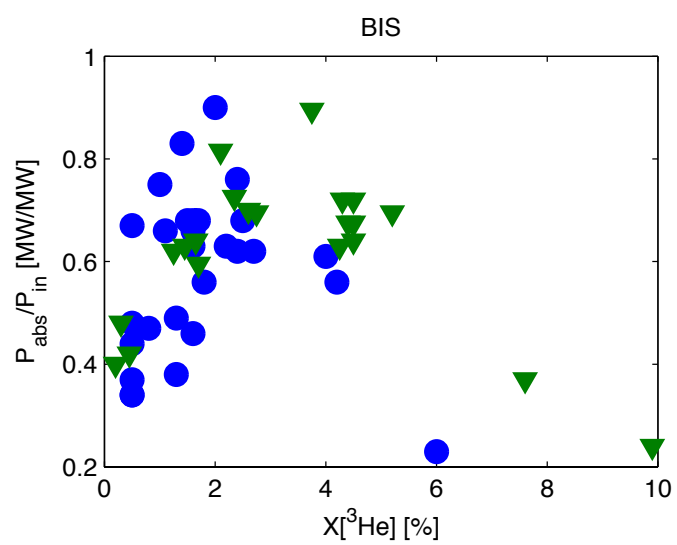

Fig. 3 : Experimental heating efficiency as function of the ${ }^{3} \mathrm{He}$ concentration as estimated during the experiment. The blue circles represent the recent results at $\mathrm{I}_{\mathrm{p}}=1.8 \mathrm{MA}$; the green triangles correspond to earlier $\left({ }^{3} \mathrm{He}\right)-\mathrm{H}$ experiments performed at $\mathrm{I}_{\mathrm{p}}=2.45 \mathrm{MA}$ [9].

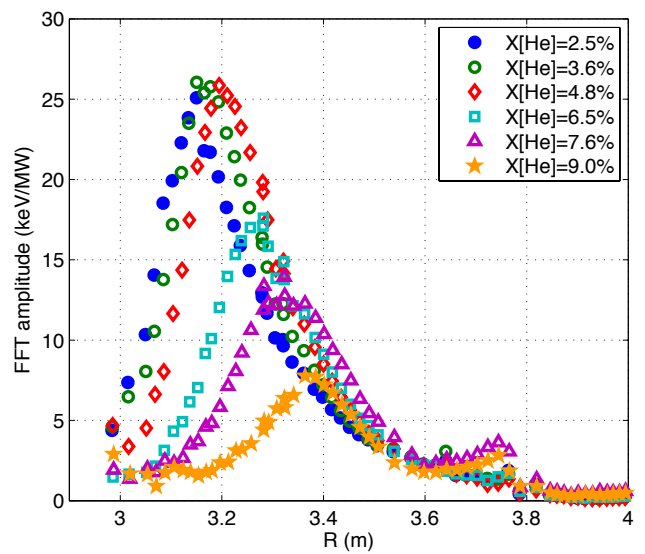

Fig. 4 : Experimental electron power deposition profiles as function of the experimentally estimated ${ }^{3} \mathrm{He}$ concentration.

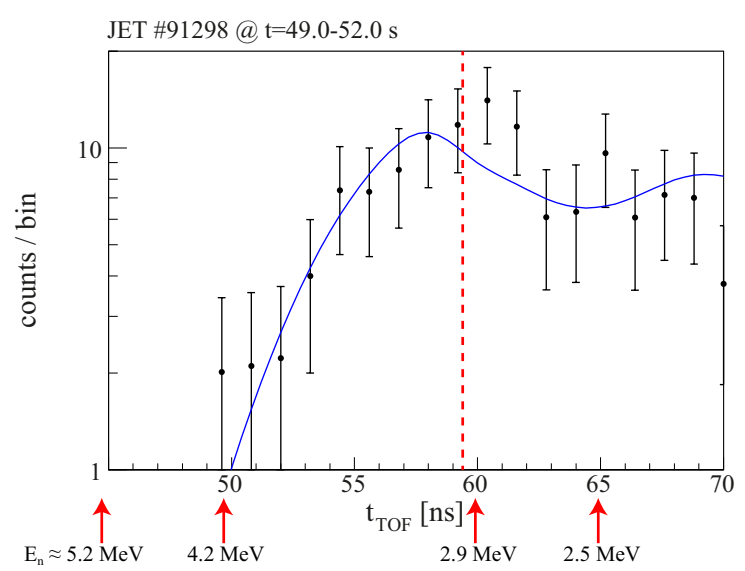

Fig. 5 : TOFOR time-of-flight neutron detector's [23] signature of RF heated deutrium minority ions. 